\title{
Systematic ranging and late warning asteroid impacts
}

\author{
D. Farnocchia ${ }^{\mathrm{a}}$, S. R. Chesley ${ }^{\mathrm{a}}$, M. Micheli ${ }^{\mathrm{b}, \mathrm{c}, \mathrm{d}}$ \\ ${ }^{a}$ Jet Propulsion Laboratory, California Institute of Technology, Pasadena, CA 91109, \\ USA \\ ${ }^{b}$ ESA NEO Coordination Centre, 00044 Frascati (RM), Italy \\ ${ }^{c}$ SpaceDyS s.r.l., 56023 Cascina (PI), Italy \\ ${ }^{d}$ INAF-IAPS, 00133 Roma (RM), Italy
}

\begin{abstract}
We describe systematic ranging, an orbit determination technique especially suitable to assess the near-term Earth impact hazard posed by newly discovered asteroids. For these late warning cases, the time interval covered by the observations is generally short, perhaps a few hours or even less, which leads to severe degeneracies in the orbit estimation process. The systematic ranging approach gets around these degeneracies by performing a raster scan in the poorly-constrained space of topocentric range and range rate, while the plane of sky position and motion are directly tied to the recorded observations. This scan allows us to identify regions corresponding to collision solutions, as well as potential impact times and locations. From the probability distribution of the observation errors, we obtain a probability distribution in the orbital space and then estimate the probability of an Earth impact. We show how this technique is effective for a number of examples, including $2008 \mathrm{TC}_{3}$ and $2014 \mathrm{AA}$, the only two asteroids to date discovered prior to impact.
\end{abstract}

Email address: Davide.Farnocchia@jpl.nasa.gov (D. Farnocchia)

Preprint 
Keywords: Asteroids, Asteroids, dynamics, Astrometry, Near-Earth objects, Orbit determination

\section{Introduction}

When an asteroid is first discovered, the few available observations rarely allow the determination of its orbit. Surveys usually capture $3-5$ repeat images of the same area of sky with a typical return time of 15-30 minutes. Over this time interval, an object in the field of view moves with respect to background stars at a roughly linear rate. Thus, the short arc of available data, also called a tracklet (Kubica et al., 2007), provides an estimate of the angular position and motion of the object in the plane of sky, which is not enough to determine a six-parameter orbit (Milani and Knežević, 2005).

Despite the degeneracies in the orbit estimation process, it is important to recognize potentially hazardous objects shortly after discovery. In fact, the only two objects discovered prior to an Earth impact, namely $2008 \mathrm{TC}_{3}$ and 2014 AA, were both discovered by R. Kowalski (Kowalski et al., 2008, 2014) of the Catalina Sky Survey (Larson et al., 1998) only about 20 hours before striking the Earth. On one hand, $2008 \mathrm{TC}_{3}$ was quickly recognized as potential impactor and so it was extensively observed before the impact. The acquired observations allowed the estimation of the orbit of $2008 \mathrm{TC}_{3}$ as well as some physical characterization (Jenniskens et al., 2009; Kozubal et al., 2011; Scheirich et al., 2010). Using the available data, it was possible to predict the impact that took place above Sudan on 2008 October t1 (Jenniskens et al.,

\footnotetext{
${ }^{1}$ http://neo.jpl.nasa.gov/news/2008tc3.html
} 
2009). On the other hand, $2014 \mathrm{AA}$ was not immediately recognized as a possible impactor and so only seven astrometric observations over about 70 minutes were obtained before the object fell into the Atlantic Ocean on 2014 January 22 (Chesley et al., 2015).

When the standard differential correction procedure (e.g., Farnocchia et al., 2015; Milani and Gronchi, 2010) to find a least-squares orbit fails, other methods can be used to assess the orbital probability distribution. In particular, Muinonen and Bowell (1993) show how to put a probability density on the phase space of the orbital elements by using Bayesian inversion theory. By using a Monte Carlo approach one can sample the orbit phase space and thereby derive the probability distribution from the observation errors corresponding to the sampled orbit.

The available observations directly constrain the position and motion of the asteroid in the sky while the distance between the asteroid and the observer (topocentric range) is poorly constrained. Thus, ranging methods are the preferred Monte Carlo approach when only a short arc of observations is available. Virtanen et al. (2001) describe a method called statistical ranging that allows one to generate orbital samples compatible with the observational data. After choosing two observations, the topocentric ranges at the epochs of the two observations are randomly sampled and a corresponding orbit computed. Oszkiewicz et al. (2009) rely on the statistical ranging technique and use Markov chains to generate an unbiased sequence of orbital samples distributed according to the probability distribution coming from Bayesian

\footnotetext{
${ }^{2}$ http://www.nasa.gov/jpl/asteroid/first-2014-asteroid-20140102
} 
inversion theory.

Chesley (2005) introduces a technique called systematic ranging3, which in contrast to Monte Carlo techniques systematically explores a raster in the topocentric range and range-rate space. This technique provides a geometric description of the orbital elements as a function of range and range rate. Moreover, systematic ranging allows one to identify regions of the phase space filled with impact solutions and the corresponding impact times and locations. In this paper we present a detailed description of systematic ranging and show how to derive a probability distribution on the range and range-rate space, which is then mapped to the orbital element space where impact probability estimates can be derived.

\section{Systematic ranging}

Systematic ranging relies on the fact that a short arc of observations yields a direct estimate of the plane of sky position (right ascension $\alpha$ and declination $\delta$ ) and motion $(\dot{\alpha}$ and $\dot{\delta})$. These four scalar quantities can be assembled

together in the so-called attributable $\mathcal{A}=(\alpha, \delta, \dot{\alpha}, \dot{\delta})$ (Milani and Knežević, 2005). The topocentric range $\rho$ and $\dot{\rho}$ are only marginally constrained, if at all. If $\rho$ and $\dot{\rho}$ were known, we would have a full description of the asteroid's topocentric position and velocity in polar coordinates $(\alpha, \delta, \dot{\alpha}, \dot{\delta}, \rho, \dot{\rho})$, which can be easily converted to a Cartesian heliocentric state if the position and velocity of the observer are known. Note that, to account for lighttime correction, we correct the epoch of the Cartesian state from that of the

\footnotetext{
${ }^{3}$ This technique was actually introduced by Tholen and Whiteley in 2002, but the paper was never published.
} 
attributable by a quantity $\rho / c$, where $c$ is the speed of light.

To explore the orbit phase space, systematic ranging scans a suitably dense grid in the $(\rho, \dot{\rho})$ space. Such grid needs to be large enough to contain the reasonably possible orbital configurations. In particular, the grid must contain the so-called admissible region (Milani et al., 2004), i.e., the values of $(\rho, \dot{\rho})$ leading to bounded heliocentric orbits. For each grid point we fix the values of $\rho=\rho_{i}$ and $\dot{\rho}=\dot{\rho}_{j}$ and find the best fit value of the attributable $\mathcal{A}_{i j}$ that minimizes the cost function:

$$
Q=\boldsymbol{\nu}^{T} W \boldsymbol{\nu}
$$

where $\boldsymbol{\nu}$ is the vector of Observed - Computed astrometric residuals and $W$ is the weight matrix (Farnocchia et al., 2015).

The constrained best-fitting solution can easily be converted to an orbit, which is in turn propagated to find upcoming Earth encounters. Moreover, if the observations contain photometric measurements we also compute the absolute magnitude for each grid point.

Figure 1/shows the application of systematic ranging to 2014 AA by using the seven astrometric observations obtained by the Catalina Sky Survey prior to impact. The orbital elements are shown as a function of $(\rho, \dot{\rho})$. The dashed curve encloses bounded orbits and corresponds to the admissible region of Milani et al. (2004). The dash-dotted line is for grazing impacts: the region on the left of the curve contains impacting solutions.

\section{Probability distribution on the range $\&$ range rate space}

The raster scan presented in the previous section gives a geometric description of how the orbital configuration depends on the value of topocentric 

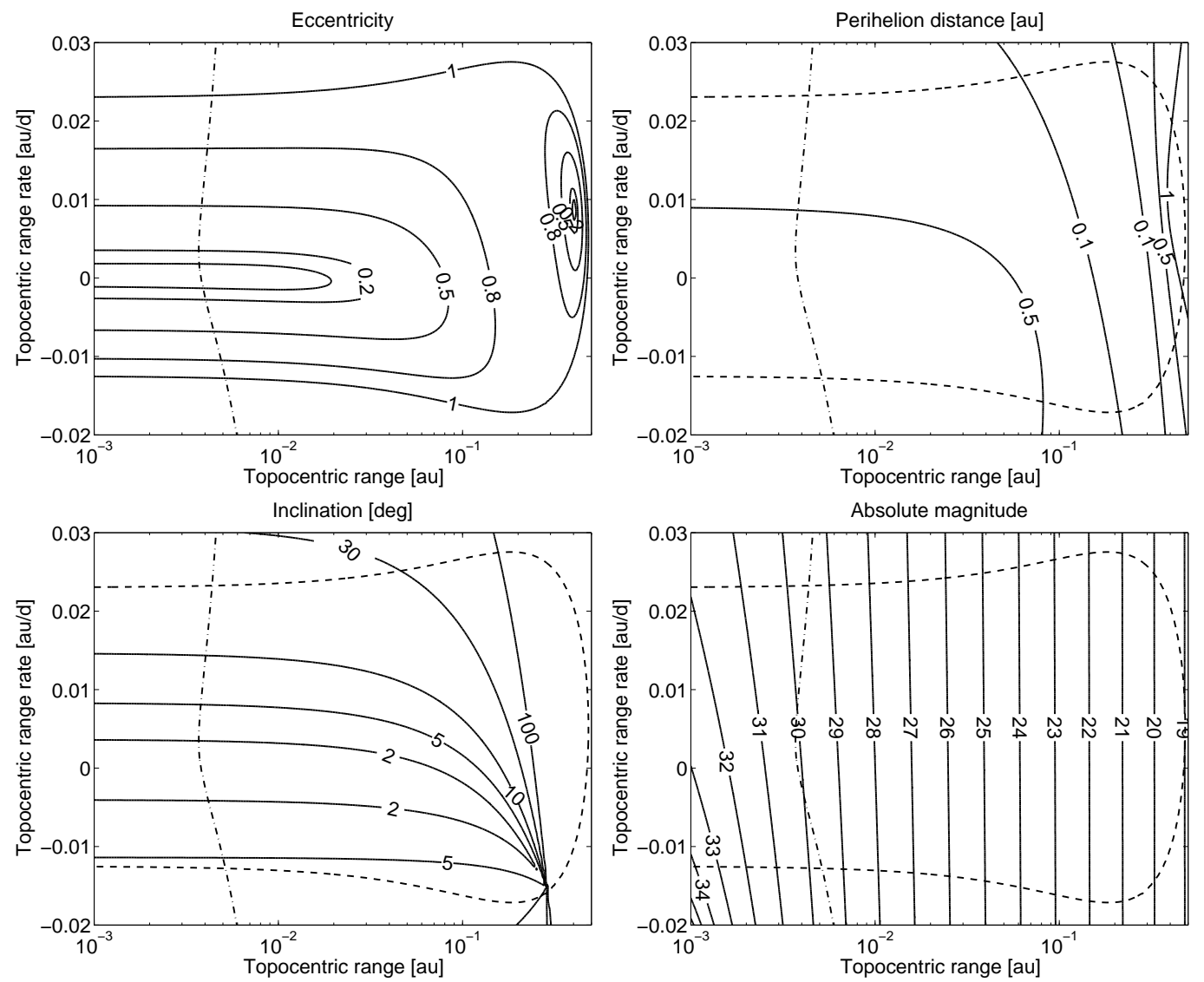

Figure 1: Eccentricity, perihelion distance, inclination, and absolute magnitude as a function of range and range rate for asteroid 2014 AA. The dashed curve corresponds to parabolic orbits and the dash-dotted line to grazing impacts. 


\begin{tabular}{|cc|cc|cc|}
\hline Station & $\sigma_{\alpha, \delta}$ & Station & $\sigma_{\alpha, \delta}$ & Station & $\sigma_{\alpha, \delta}$ \\
\hline 703 & $1.00^{\prime \prime}$ & G96 & $0.50^{\prime \prime}$ & $\mathrm{F} 51$ & $0.20^{\prime \prime}$ \\
950 & $0.50^{\prime \prime}$ & 291 & $0.70^{\prime \prime}$ & 691 & $0.70^{\prime \prime}$ \\
568 & $0.15^{\prime \prime}$ & $\mathrm{H} 01$ & $0.30^{\prime \prime}$ & $\mathrm{H} 21$ & $0.70^{\prime \prime}$ \\
$\mathrm{J} 04$ & $0.40^{\prime \prime}$ & $\mathrm{W} 84$ & $0.20^{\prime \prime}$ & $\mathrm{F} 65, \mathrm{E} 10$ & $0.40^{\prime \prime}$ \\
W85, W86, W87 & $0.60^{\prime \prime}$ & $\mathrm{Q} 63, \mathrm{Q} 64, \mathrm{~V} 37$ & $0.80^{\prime \prime}$ & $\mathrm{K} 91, \mathrm{~K} 92, \mathrm{~K} 93$ & $0.80^{\prime \prime}$ \\
\hline
\end{tabular}

Table 1: Station-specific weighting rules for the most productive discovery and short-term follow-up stations. For all the other stations we use weights at $1^{\prime \prime}$.

range and range rate. The next step is to assign a probability distribution to this space.

As is common practice, given a set of optical observations we assume that the observation errors $\boldsymbol{\nu}$ are normally distributed according to a weight matrix $W$, i.e., their probability density $f_{\text {err }}$ is:

$$
f_{\text {err }}(\boldsymbol{\nu}) \propto \exp \left(-0.5 \boldsymbol{\nu}^{T} W \boldsymbol{\nu}\right)
$$

It is typical to use a diagonal weight matrix where the individual weights are $1 / \sigma^{2}$ ( $\sigma$ is the uncertainty in the astrometric positions). Table 1 shows the data weights we currently use for the most productive discovery and followup stations. The chosen weights are based on our experience and account for the fact that discovery observations can sometimes be problematic because they are not targeted on the object.

In principle, it is possible to consider the photometric residuals together with the astrometric ones. However, photometric measurements have a much larger uncertainty than that of the astrometry and can be affected by signifi- 
cant biases (Jurić et al., 2002). Moreover, in case of a magnitude trend, it is hard to tell if the varying luminosity is due to a rapidly changing topocentric distance rather than the asteroid's rotation. Therefore, we do not use the information obtained from the photometric residuals.

According to Bayesian inversion theory (Muinonen and Bowell, 1993), the posterior probability density function for $(\rho, \dot{\rho})$ is:

$$
f_{\text {post }}(\rho, \dot{\rho}) \propto f_{\text {err }}(\boldsymbol{\nu}(\rho, \dot{\rho})) f_{\text {prior }}(\rho, \dot{\rho})
$$

where $f_{\text {prior }}$ is a prior distribution on the $(\rho, \dot{\rho})$ space 4 The selection of $f_{\text {prior }}$ is to some extent arbitrary and far from trivial (Jaynes, 1968), and yet can significantly affect the posterior probability distribution, especially for short observation arcs.

Whatever the choice of $f_{\text {prior }}$, we always add a crude constraint from the population model by setting $f_{\text {prior }}=0$ for hyperbolic orbits, flagging objects where the astrometric errors favor an unbounded orbit.

\subsection{Jeffreys' prior}

A mathematically sound choice is Jeffreys' prior (Granvik et al., 2009):

$$
f_{\text {prior }}(\rho, \dot{\rho})=\sqrt{\operatorname{det}\left(\frac{\partial \boldsymbol{\nu}}{\partial(\rho, \dot{\rho})}^{T} W \frac{\partial \boldsymbol{\nu}}{\partial(\rho, \dot{\rho})}\right)} .
$$

Note that the partials are the total derivatives of $\boldsymbol{\nu}$ with respect to $(\rho, \dot{\rho})$, which means that they account for the fact that the best-fit attributable $\mathcal{A}$ changes as a function of $(\rho, \dot{\rho})$.

\footnotetext{
${ }^{4}$ It is often convenient to use a logarithmic scale for $\rho$ to achieve a better resolution at small topocentric distances. In that case the probability density has to be multiplied by $\rho$.
} 
Among other things, Jeffreys' prior secures the invariance of the probability distribution when changing variables. However, since there is more sensitivity of the residuals for small topocentric distances, Jeffreys' prior tends to favor orbital configurations where the object is close to the observer, e.g., see the examples of $2015 \mathrm{CV}$ and $2015 \mathrm{BU}_{92}$ in Sec. 6.

\subsection{Prior based on population model}

As already discussed by Virtanen et al. (2001), the prior could be based on the known asteroid population. Even better, one could use an orbit and size population model where the known population is extrapolated to remove survey selection effects and to reach completeness up to a given apparent magnitude. One such model is the Pan-STARRS Synthetic Solar System Model (Grav et al., 2011), and new population models are expected in the future (Granvik et al., 2014). From the Grav et al. (2011) model we derived a probability density $f_{\text {pop }}(q, e, i, H)$ for perihelion distance $q$, eccentricity $e$, inclination $i$, and absolute magnitude $H$. A more refined approach would be to remove the known population from the population model.

Mapping $f_{\text {pop }}$ to the best fitting orbits in the $(\rho, \dot{\rho})$ plane is quite complicated. Therefore, to compute such a prior we iteratively apply Bayesian inversion theory, i.e., the prior is computed as posterior $f_{\text {post }}^{\prime}$ of another prior $f_{\text {prior }}^{\prime}$

$$
f_{\text {prior }}(\rho, \dot{\rho})=f_{\text {post }}^{\prime}(\rho, \dot{\rho}) \propto f_{\text {prior }}^{\prime}(\rho, \dot{\rho}) f_{\text {pop }}(q(\rho, \dot{\rho}), e(\rho, \dot{\rho}), i(\rho, \dot{\rho}), H(\rho, \dot{\rho})) \text {. }
$$

At this point we select $f_{\text {prior }}^{\prime}$ using simple geometric considerations. By assuming that the Cartesian space of position and velocity is uniformly filled, we have that $\rho^{3}$ and $\dot{\rho}$ are uniformly distributed. Hence, $f_{\text {prior }}^{\prime}(\rho, \dot{\rho}) \propto \rho^{2}$. 


\subsection{Uniform prior}

A common choice for $f_{\text {prior }}$ is a uniform distribution. For instance, such a prior is used by Virtanen et al. (2001) and Oszkiewicz et al. (2009). Interestingly, it is possible to approximately obtain a prior distribution from the population model if we only consider the size distribution $f_{\text {size }}(H)$, i.e., $f_{\text {prior }}=\rho^{2} f_{\text {size }}(H)$.

For near-Earth asteroids the cumulative size distribution is expected to follow a power law $f_{\text {size }}(H) \propto 10^{\eta H}$, with $\eta$ somewhere between 0.35 and 0.47 (Bottke et al., 2002; Harris, 2002; Stuart and Binzel, 2004). For a given visual magnitude $V$, we have that $f_{\text {size }}(H) \propto \rho^{-5 \eta}$ (Bowell et al., 1989) and

therefore $f_{\text {prior }}=\rho^{2-5 \eta}$. According to the range of possible values for $\eta$, this prior corresponds to a power law with exponent between -0.35 and 0.25 , which is compatible with a uniform distribution.

Though a uniform distribution might appear overly simplistic, we have found that this it does a good job of identifying potential impactors (e.g., see Sec. 6) and distinguishing interesting objects from more common ones, such as main belt asteroids.

\section{Impact probability computation}

For each grid point, as a result of the constrained least squares fit to the observations, we obtain not only a best fit value $\mathcal{A}_{i j}$ of the attributable, but also the corresponding uncertainty expressed by a covariance matrix $\Gamma_{\mathcal{A}_{i j}}$. This uncertainty is easily mapped to the orbital element space and therefore allows us to compute the probability $p_{i j}$ of an Earth impact for that orbit. Since for a fixed grid point the orbital uncertainty is small and the horizon 
for impacts is of the order of weeks, we compute the impact probability with a linear approach (Milani et al., 2002; Farnocchia et al., 2015).

Given $f_{\text {post }}$ we can assign each grid point a weight $w_{i j} \propto f_{\text {post }}\left(\rho_{i}, \dot{\rho}_{j}\right)$, where $\sum w_{i j}=1$. Then, the impact probability can be easily computed as

$$
P=\sum w_{i j} p_{i j}
$$

\section{Generation of Monte Carlo orbital samples}

To better visualize the orbital distribution, it is convenient to generate Monte Carlo orbital samples distributed according to the probability distribution computed above. The following procedure can be used to achieve this goal:

(a) Randomly draw $\rho_{k}$ according to a uniform distribution between the minimum and maximum values of $\rho$ on the grid;

(b) Randomly draw $\dot{\rho}_{k}$ according to a uniform distribution between the minimum and maximum values of $\dot{\rho}$ on the grid;

(c) Randomly draw a value $\gamma$ according to a uniform distribution between 0 and the maximum of $f_{\text {post }}$ on the grid;

(d) Compute a best-fit attributable $\mathcal{A}_{k}$ for $(\rho, \dot{\rho})=\left(\rho_{k}, \dot{\rho}_{k}\right)$;

(e) If $f_{\text {post }}\left(\rho_{k}, \dot{\rho}_{k}\right) \geq \gamma$ go to (f), otherwise start again from (a);

(f) Add normally distributed noise to $\mathcal{A}_{k}$ according to the covariance $\Gamma_{\mathcal{A}_{k}}$ and add $\left(\mathcal{A}_{k}, \rho_{k}, \dot{\rho}_{k}\right)$ to the sequence of orbital samples. 

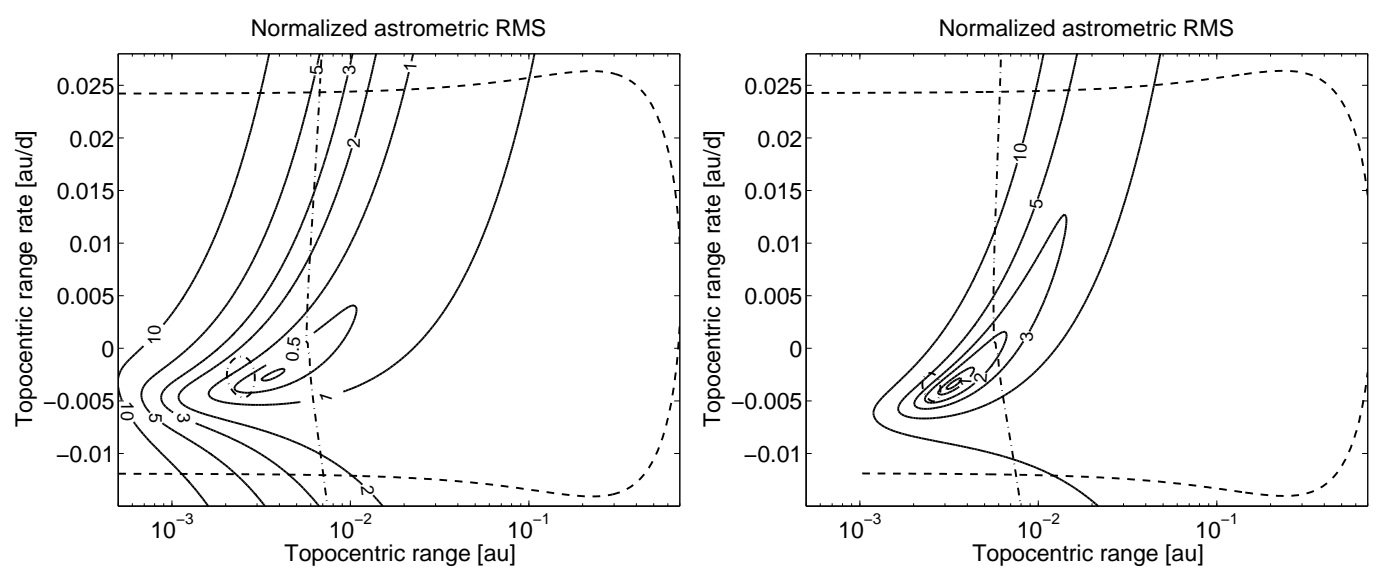

Figure 2: Normalized RMS as a function of $(\rho, \dot{\rho})$ for $2008 \mathrm{TC}_{3}$. Left panel is for the first tracklet (four observations over $43 \mathrm{~min}$ ), right panel is for the first two tracklets (total of seven observations over $99 \mathrm{~min})$. Astrometric weights are at $0.5^{\prime \prime}$. The dashed curve corresponds to parabolic heliocentric orbits and the dash-dotted line to grazing impacts.

\section{Examples}

We want to test the algorithm presented in the previous sections on real cases. On one hand it is important that systematic ranging provides an early detection of impacting asteroids. On the other hand we need to avoid generating too many warnings for objects that might be very far from Earth. Thus, we show examples of impactors as well as main belt asteroids. Table 2 summarizes the results for the examples discussed in more detail in the following subsections.

\section{1. $2008 T C_{3}$}

As already discussed in the introduction, $2008 \mathrm{TC}_{3}$ was the first asteroid discovered prior to impact. This object was first spotted and promptly followed up by R. A. Kowalski at the Mt. Lemmon observatory. In the 
20 hours between discovery and impact hundreds of astrometric observations were reported to the Minor Planet Center. These observations allowed the calculation of a least squares orbit and the prediction of the impact. How would systematic ranging have performed with the first observations of $2008 \mathrm{TC}_{3}$ ?

The left panel of Fig. 2 shows the normalized RMS, i.e., the square root of the target function $Q$ in Eq. (11), as a function of $(\rho, \dot{\rho})$ by using the first tracklet of four observations obtained by Kowalski. The nonlinearity of the orbit determination problem is evident from the level curves of the RMS, which clearly suggest that $2008 \mathrm{TC}_{3}$ could be on an impact trajectory. As a matter of fact, the corresponding impact probability, computed as described in Sec. 4, is already significant with the first four observations (Table 2). With our defaults data weights at $0.5^{\prime \prime}$, the impact probability is few percents when using a uniform prior or one based on the population model, and $\sim 60 \%$ when using Jeffreys' prior. With more conservative weights at $1^{\prime \prime}$ Jeffreys' prior leads to a $\sim 70 \%$ impact probability, while the uniform and population prior result in a probability of few in a thousand.

With the addition of Kowalski's second tracklet, three additional observations, the orbit becomes much better constrained by the observation residuals (see right panel of Fig. 2). The impact is now almost certain, especially with $0.5^{\prime \prime}$ weights.

By using the first two tracklets of data and adopting a uniform prior, we also generated Monte Carlo samples to find potential impact locations, as shown in Fig. 3. As expectable, the region of possible impact location contains the Sudan desert where $2008 \mathrm{TC}_{3}$ actually ended its journey 


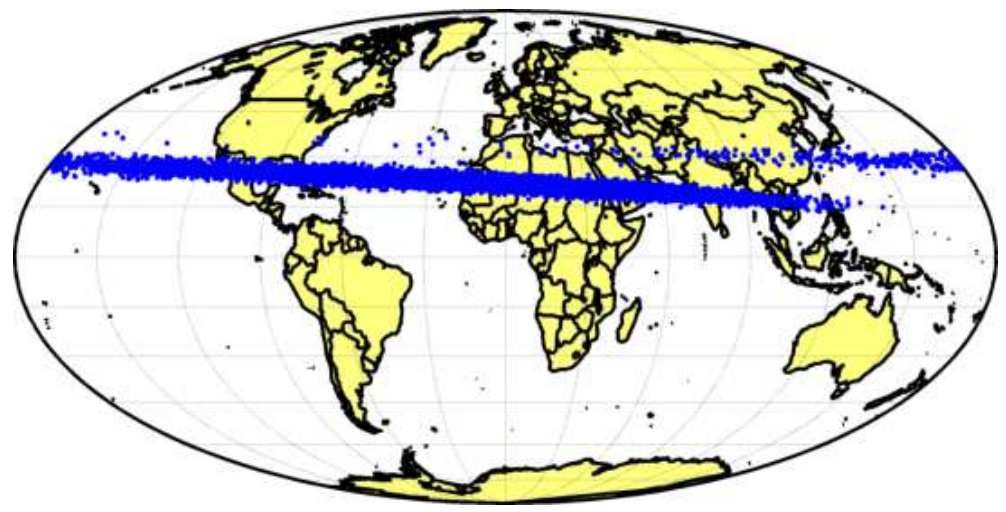

Figure 3: Potential impact locations for $2008 \mathrm{TC}_{3}$ based on the first two tracklets of data.

(Jenniskens et al., 2009).

\section{2. $2014 A A$}

Though 2014 AA was not extensively followed up after discovery, there are several similarities with $2008 \mathrm{TC}_{3}$. In particular, $2014 \mathrm{AA}$ was also discovered about 20 hours before impact by R. A. Kowalski at the Mt. Lemmon observatory.

Figure 4 shows the normalized RMS for 2014 AA as a function of $(\rho, \dot{\rho})$ by using the first tracklet of data (three observations) or the full available dataset (seven observations). Again, the RMS curves clearly indicate that an impact is possible. Systematic ranging immediately recognizes the potential impact from the first tracklet and make the impact almost certain when the second tracklet is added. Table 2 reports the computed impact probabilities, which are similar to those computed for $2008 \mathrm{TC}_{3}$,

The impact locations (see Fig. [5) found by using the seven available observations and adopting a uniform prior are statistically consistent with the detection by the infrasound component of the International Monitoring Sys- 

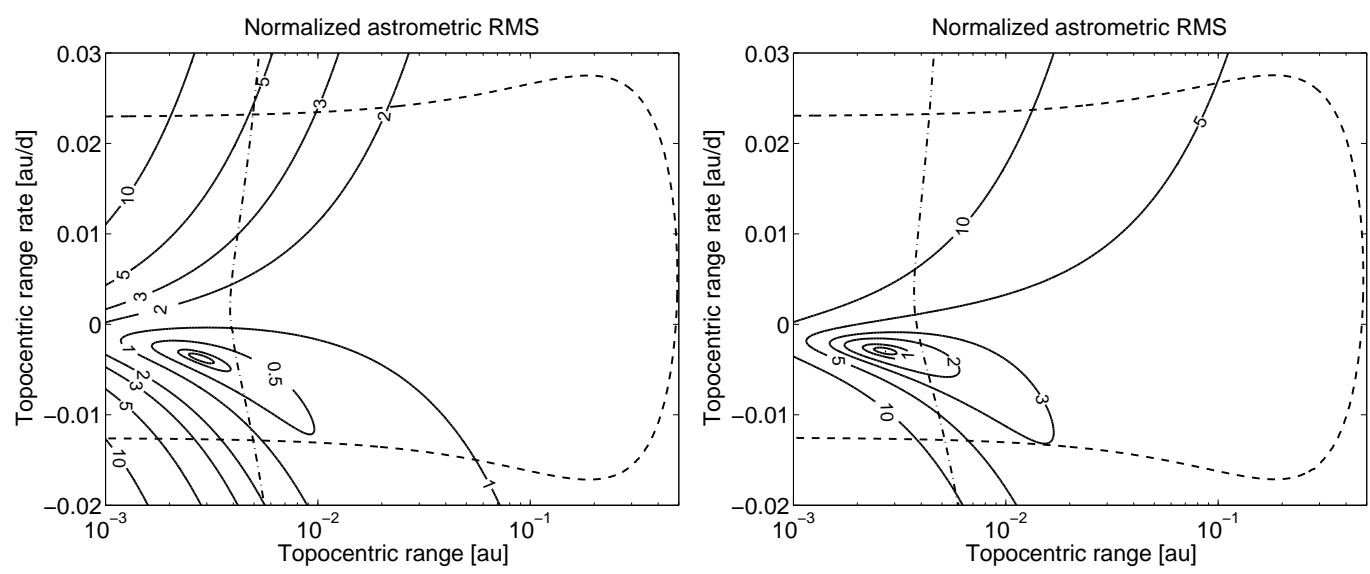

Figure 4: Normalized RMS as a function of $(\rho, \dot{\rho})$ for 2014 AA. Left panel is for the first tracklet (three observations over $28 \mathrm{~min}$ ), right panel is for the first two tracklets (total of seven observations over $69 \mathrm{~min})$. Astrometric weights are at $0.5^{\prime \prime}$. The dashed curve corresponds to parabolic heliocentric orbits and the dash-dotted line to grazing impacts.

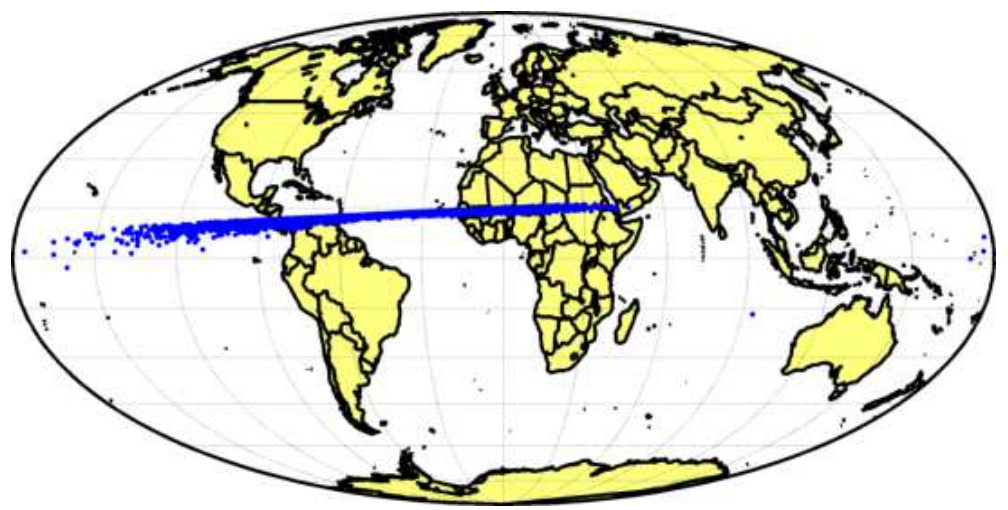

Figure 5: Potential impact locations for 2014 AA based on all available data, i.e., seven observations. 

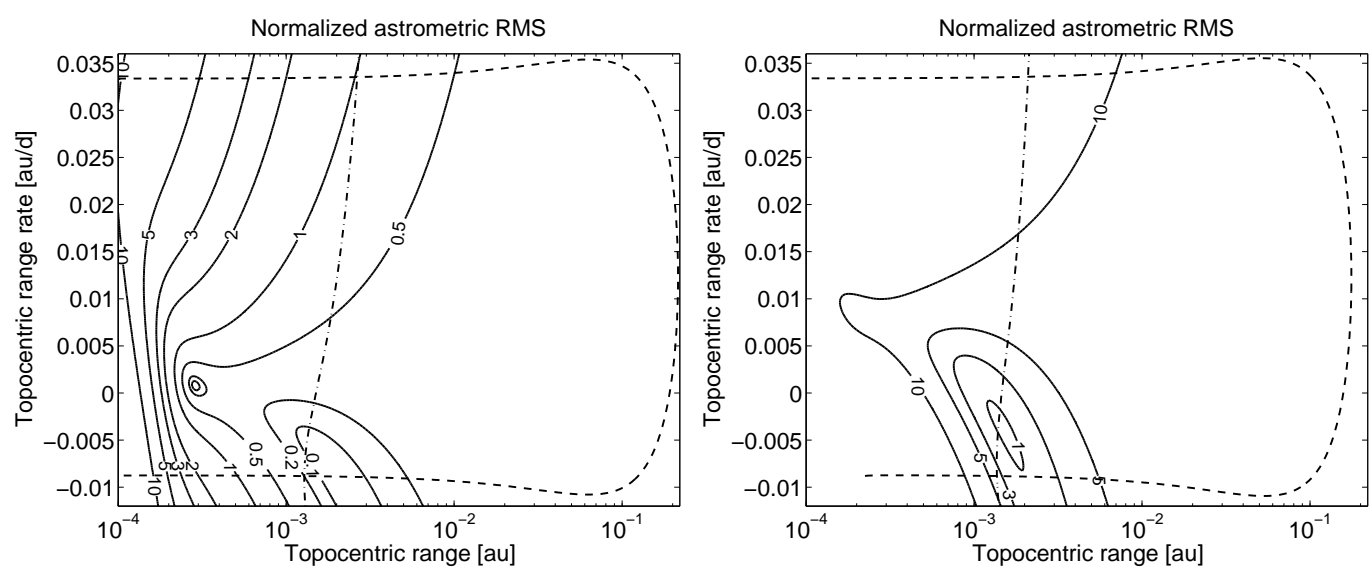

Figure 6: Normalized RMS as a function of $(\rho, \dot{\rho})$ for Hayabusa. Left panel is for the first tracklet (three observations over $7 \mathrm{~min}$ ), right panel is for the first two tracklets (total of seven observations over $34 \mathrm{~min}$ ). Astrometric weights are at $1.0^{\prime \prime}$. The dashed curve corresponds to parabolic heliocentric orbits and the dash-dotted line to grazing impacts.

tem (IMS) operated by the Comprehensive Nuclear Test Ban Treaty Organization (CTBTO), which places the impact location in the Atlantic Ocean at a latitude of about $15^{\circ} \mathrm{N}$ and a longitude of about $43^{\circ} \mathrm{W}$ (Chesley et al., 2015; Brown, 2014).

\subsection{Hayabusa \& Hayabusa 2}

Because of the low number of actual impactors we can use as a validation, we tested our algorithm on Hayabusa and Hayabusa 2 (Kawaguchi et al., 2008).

The reentry of Hayabusa took place on 2010 June 13. Yamaguchi et al. (2011) report ground-based observations acquired during the reentry phase, which ended with a landing in the South Australia outback. We used these observations for applying systematic ranging technique to Hayabusa. Figure 6 shows the normalized RMS as a function of $(\rho, \dot{\rho})$ by using the first 
tracklet of data (three observations) or the full available dataset (six observations). Table 2 gives the impact probability as a function of the number of observations. With a uniform prior the initial impact probability is $2.7 \times 10^{-3}$ and increases to $17 \%$ with the second tracklet. With the population-based prior the initial impact probability is $2.0 \times 10^{-3}$ and increases to $2 \%$ with the second tracklet. On the other hand, with the use of Jefrreys' prior we immediately have a $94 \%$ impact probability that decreases to $24 \%$ with the second tracklet. This counterintuitive behavior is due to the presence of two local RMS minima with the first tracklet, one of which clearly corresponds to an impact and is ruled out by the second tracklet.

Hayabusa 2 was launched on 2014 December 3.5 After launch Hayabusa 2 was initially put on a low Earth parking orbit, until a maneuver moved it onto a ballistic trajectory leaving the Earth. On its way out, on 2014 December 6, Hayabusa 2 happend to be in the field of view of the Pan-STARRS PS1 survey (Wainscoat et al., 2014), which collected three observations of the spacecraft over $43 \mathrm{~min}$. The corresponding astrometry was submitted to the Minor Planet Center, which, since the observations were not recognized as belonging to the Hayabusa 2 spacecraft, put the object on the NEO Confirmation Page 6 with the temporary designation P10gJfF. The following day, 2014 December 7, Pan-STARRS obtained three additional observations from which it became clear that the object was Hayabusa 2. Table 2 shows how systematic ranging recognizes Hayabusa 2 as potential impactor from the first three observations with an impact probability probability of few to ten percents, depending on

\footnotetext{
${ }^{5}$ http://global.jaxa.jp/press/2014/12/20141203_h2af26.html

${ }^{6} \mathrm{http}: / /$ www.minorplanetcenter.net/iau/NEO/ToConfirm.html
} 
the data weights and the prior used. Then, the subsequent observations better constrain the orbit and lower the impact probability since the ballistic trajectory does not correspond to an impact but to the post-injection escape orbit.

\subsection{4 $A S_{1}$}

Asteroid $2004 \mathrm{AS}_{1}$ was discovered by LINEAR (Stokes et al., 2000) on 2004 January 13 (Blythe et al., 2004a). The four discovery observations span $71 \mathrm{~min}$ and show an unusually high curvature thus suggesting that $2004 \mathrm{AS}_{1}$ was close to the Earth and that an impact was possible. Follow-up observations later showed that the object was much farther than originally thought, thus ruling out any impact, and that the observed curvature was due to large astrometric errors in the discovery observations.

Virtanen and Muinonen (2006) employed the statistical ranging technique (Virtanen et al., 2001) to analyze the $2004 \mathrm{AS}_{1}$ case. They found an $18 \%$ impact probability with $1.0^{\prime \prime}$ weights and concluded that such a large impact probability was mostly due to the poor quality of the discovery night observations.

The left panel of Fig. 7 shows the application of systematic ranging to $2004 \mathrm{AS}_{1}$. The astrometric observations are weighted at $1.0^{\prime \prime}$. The RMS favors an impact solution while the true solution (marked with a cross) correspond to larger astrometric errors. The impact probabilities computed for the different choices of the prior distribution are of the order of $10 \%$, see Table 2, Decreasing the weights at $1.5^{\prime \prime}$ gives significantly lower probabilities for the uniform and population-based priors, while with Jefrreys' prior we still have a $12 \%$ impact probability. 

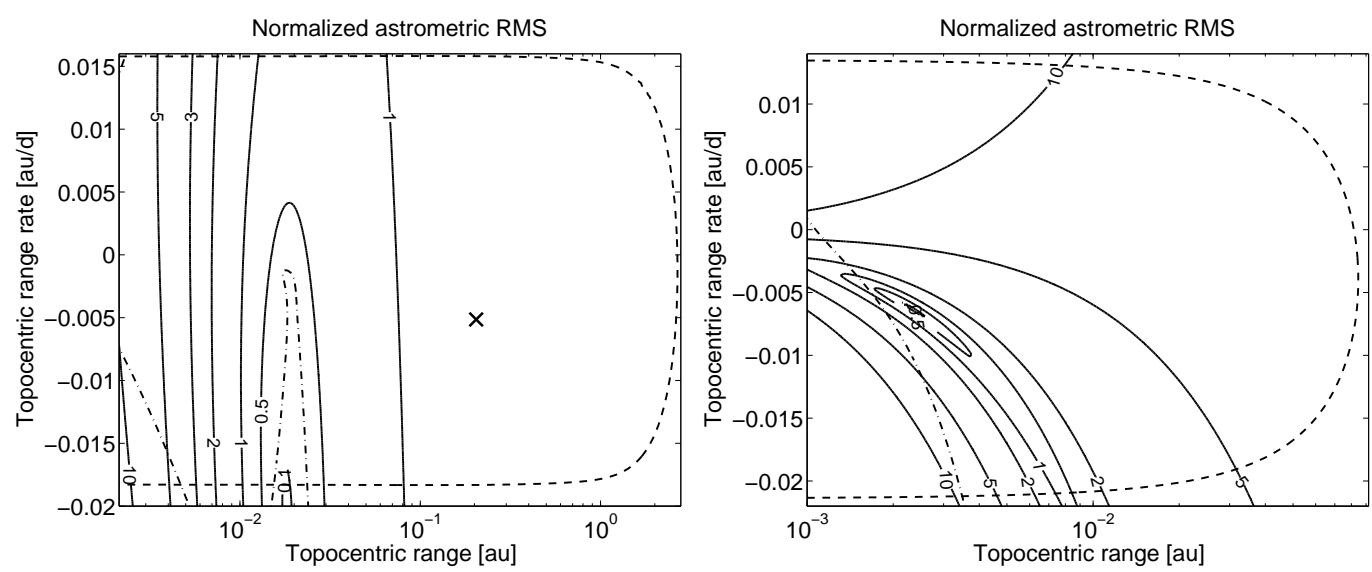

Figure 7: Normalized RMS as a function of $(\rho, \dot{\rho})$ for $2004 \mathrm{AS}_{1}$ (left panel) and $2004 \mathrm{FU}_{162}$ (right panel). Astrometric weights are at 1.0". The dashed curve corresponds to parabolic heliocentric orbits and the dash-dotted line to grazing impacts. For $2004 \mathrm{AS}_{1}$ the cross marks the true solution.

As shown by Table 3, we computed the $p$-value of the true solution, i.e., the fraction of less likely orbital solutions, for the different choices of the prior and different data weights. Jeffreys' prior produces very small $p$-values thus indicating that the true solution is deemed as extremely unlikely by the computed probability distribution. For the uniform distribution we have $3 \%$ $p$-value with $1^{\prime \prime}$ weights, which is due to the large errors in the astrometric observations. As a matter of fact, relaxing the weights increases the $p$-value to $22 \%$. On the other hand, the population-based prior compensates for the large astrometric errors since impacting solutions with low RMS are less likely in the population model than non-impacting solutions with a higher RMS. The $p$-values are $13.6 \%$ and $38.7 \%$ depending on the data weights.

The conclusion fpr $2004 \mathrm{AS}_{1}$ is that the discovery tracklet, weighted at $1^{\prime \prime}$, yields impact probabilities $\sim 10 \%$ even as the trueth is compatible with 
the data at the $3-14 \%$ level. This outcome is a manifestation of the large astrometric errors in the data.

\section{5. $2004 F U_{162}$}

Asteroid $2004 \mathrm{FU}_{162}$ was also discovered by LINEAR on 2004 March 31 (Blythe et al., 2004b). Chesley (2005) first analyzed this object and estimated that there was a $0.3 \%$ probability of an impact and that the object was likely to pass within 10 Earth radii.

The right panel of Fig. 7 shows our implementation of systematic ranging, while Table 2 reports the impact probabilities for different weights and prior distributions. Again, Jeffreys' prior results in very high impact probabilities while the population-based prior gives the lowest impact chances. For $1^{\prime \prime}$ weights the impact probability is $0.7 \%-0.9 \%$ with the uniform and the population based priors. An Earth encounter within 10 Earth radii is assured with all three priors, thus confirming the findings of Chesley (2005).

\section{6. $2015 C V \& 2015 B U_{92}$}

The previous examples show how systematic ranging is capable of quickly identifying actual and potential impactors. However, there is the risk of having significant impact probabilities $\left(10^{-3}\right.$ or higher $)$ for a large number of objects, which would make it difficult to distinguish interesting objects from less remarkable ones. Here we show the example of two main belt objects that were recently posted on the NEO Confirmation Page when the initial observations were compatible with an NEO orbit: $2015 \mathrm{CV}$ and $2015 \mathrm{BU}_{92}$.

2015 CV was discovered by the Catalina Sky survey, Mt. Lemmon station, on 2015 February 10, while 2015 BU $_{92}$ was discovered by Spacewatch 

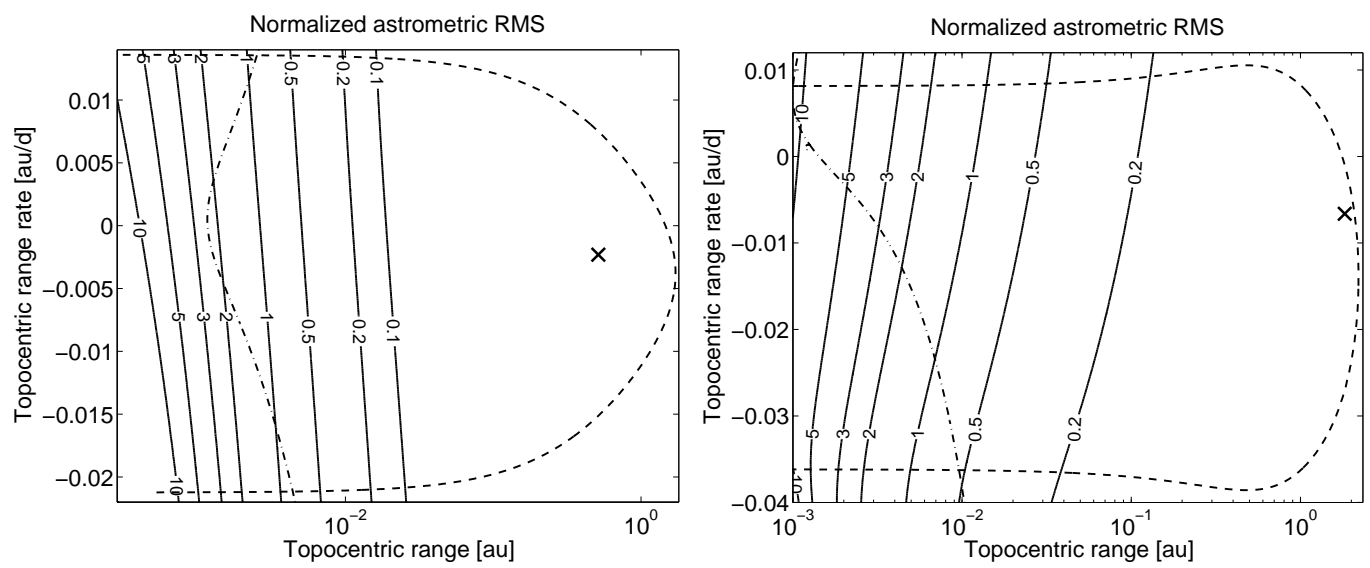

Figure 8: Normalized RMS as a function of $(\rho, \dot{\rho})$ for $2015 \mathrm{CV}$ (left panel) and $2015 \mathrm{BU}_{92}$ (right panel). Astrometric weights are at $0.5^{\prime \prime}$. The dashed curve corresponds to parabolic heliocentric orbits and the dash-dotted line to grazing impacts. Crosses mark the true solutions.

(McMillan, 2000), Kitt Peak observatory, on 2015 January 16 (Bressi and Tubbiolo, 2015). Figure 8 shows the application of systematic ranging to these two asteroids. For both objects the region with reasonable astrometric errors extends from impacting solutions to more than 1 au from the observer.

As shown by Table 2 and Table 3 , in both cases Jeffreys' prior provides significant impact probabilities and extremely low $p$-values for the true solutions. In fact, Jeffreys' prior is proportional to the partials of the astrometric residuals and the residuals do not change much for large topocentric distances while they are very sensitive when the object is close to the observer. Therefore, Jeffreys' prior tends to favor small topocentric distances even if the astrometric errors are larger. On the other hand the uniform and the population-based priors give much more reasonable probabilities and $p$ values. 


\section{Discussion and future work}

Small asteroids are expected to strike the Earth on a regular basis. For instance, according to Brown et al. (2002) impacts with $10 \mathrm{~m}$ sized objects take place every few years. Since such small asteroids are likely to be observed, if at all, only shortly before impact, it is important to be capable of an early recognition of hazardous objects.

The shortness of the observation arcs typically available for these objects leads to critical degeneracies in the orbit estimation process and limits

the applicability of the standard impact monitoring systems (Milani et al., 2005). The systematic ranging technique presented in this paper relies on Bayesian inversion theory, which overcomes these degeneracies and results in a posterior distribution on the orbit phase space.

A critical point is the choice of a prior distribution for the poorly constrained space of topocentric range and range rate. This choice is far from trivial and indeed is somewhat arbitrary. We analyzed three options: Jeffreys' prior, a uniform prior, and a prior distribution based on the asteroid population model.

Jeffreys' prior does a good job at assigning high impact probabilities to actual impactors. However, this prior produces high impact probabilities also for less remarkable objects such as main belt asteroids. The reason for this behavior is that Jeffreys' prior favors regions in the phase space that are closer to the observer. As a matter of fact, Jeffreys' prior is proportional to the partials of the residuals with respect to topocentric range and range rate, which are large for small topocentric distances and vanish at large distances. Moreover, in the non-impacting examples we analyzed we found that the true 
solution was often deemed as extremely unlikely by the posterior distribution obtained starting from Jeffreys' prior. Therefore, we avoid the use of Jeffreys' prior for systematic ranging.

The prior based on the asteroid orbit and size distribution represents the highest fidelity and most complex choice. However, it is worth pointing out that a population model is necessarily limited by the finite number of samples and is therefore affected by Poisson statistics errors. In particular, small impactors might have peculiar orbits that are deemed unlikely by the model, which could be too much of a risk when the main goal is to identify potential impactors.

A uniform distribution represents a compromise between simplicity and reliability. Though the corresponding posterior distribution and impact probabilities are not as rigorous as the one resulting from the population-based prior, we found that a uniform prior is effective at flagging potential impactors and discriminating between hazardous asteroids and more distant ones, which is the most important task to be achieved by systematic ranging. Another advantage of the uniform prior is that it has little prejudice on the actual orbit.

To complement the standard impact monitoring performed by JPL's Sentry 7 and the University of Pisa's NEODyS 8 we have implemented an automated system to continually analyze the objects on the Minor Planet Center's NEO Confirmation Page with systematic ranging. This system quickly computes an impact probability to identify potential impactors and notifies us

\footnotetext{
${ }^{7}$ http://neo.jpl.nasa.gov/risk

${ }^{8} \mathrm{http}: / /$ newton.dm.unipi.it/neodys
} 
of interesting results. In such cases, we have communicated with discoverers and other observers to solicitate a quality control of the reported astrometry and for acquiring follow-up observations, which are necessary to decrease the orbital uncertainties and conclusively determine if the impact is going to take place. The examples of $2008 \mathrm{TC}_{3}$ and $2014 \mathrm{AA}$, i.e., the only two asteroids discovered before striking the Earth, suggest that objects with an impact probability above $10^{-3}$ should be prioritized for prompt follow-up. In recent experience this happens a few to several times a month.

Among the possible additional applications of the systematic ranging technique presented in this paper, one could be the identification potential radar targets or mission-accessible targets, e.g., for the NASA's Asteroid Redirect Mission (Abell et al., 2014).

\section{Acknowledgments}

We thank T. B. Spahr for his advice on the astrometric weights of Table1.

Part of this research was conducted at the Jet Propulsion Laboratory, California Institute of Technology, under a contract with NASA.

Copyright 2015 California Institute of Technology.

\section{References}

Abell, P., Gates, M., Johnson, L., Chodas, P., Brophy, J., Mazanek, D., Muirhead, B. 2014. NASA's Asteroid Redirect Mission: Overview and Status. 40th COSPAR Scientific Assembly 40, 17.

Blythe, M, Shelly, F., Bezpalko, M., et al. 2004. 2004 AS1. Minor Planet Electronic Circulars 2004-A56. 
Blythe, M, Shelly, F., Bezpalko, M., et al. 2004. 2004 FU162. Minor Planet Electronic Circulars 2004-Q22.

Bottke, W. F., Morbidelli, A., Jedicke, R., Petit, J.-M., Levison, H. F., Michel, P., Metcalfe, T. S. 2002. Debiased Orbital and Absolute Magnitude Distribution of the Near-Earth Objects. Icarus 156, 399-433.

Bowell, E., Hapke, B., Domingue, D., Lumme, K., Peltoniemi, J., Harris, A. W. 1989. Application of photometric models to asteroids. Asteroids II 524-556.

Bressi, T. H., Tubbiolo, A. F., 2015. 2015 BU92. Minor Planet Electronic Circulars 2015-B95.

Brown, P., Spalding, R. E., ReVelle, D. O., Tagliaferri, E., Worden, S. P. 2002. The flux of small near-Earth objects colliding with the Earth. Nature 420, 294-296.

Brown, P. 2014. Using the international monitoring system of seismic, infrasound, and hydroacoustic sensors for global airburst detection. Asteroids, Comets, Meteors 201463.

Chesley, S. R. 2005. Very short arc orbit determination: the case of asteroid $2004 \mathrm{FU}_{162}$. IAU Colloq. 197: Dynamics of Populations of Planetary Systems 255-258.

Chesley, S. R., Farnocchia, D., Brown, P., Chodas, P. W. 2015. Orbit Estimation for Late Warning Asteroid Impacts: The Case of 2014 AA. IEEE Aerospace Conference, \#2.1202. 
Farnocchia, D., Chesley, S. R., Milani, A., Gronchi, G. F., Chodas, P. W. 2015. Orbits, long-term predictions, impact monitoring. Asteroids IV, In press.

Granvik, M., Virtanen, J., Oszkiewicz, D., Muinonen, K. 2009. OpenOrb: Open-source asteroid orbit computation software including statistical ranging. Meteoritics and Planetary Science 44, 1853-1861.

Granvik, M., Morbidelli, A., Jecicke, R., et al. 2014. Unbiased dynamical and physical characteristics of the near-Earth-object population. Asteroids, Comets, Meteors 2014180.

Grav, T., Jedicke, R., Denneau, L., Chesley, S., Holman, M. J., Spahr, T. B. 2011. The Pan-STARRS Synthetic Solar System Model: A Tool for Testing and Efficiency Determination of the Moving Object Processing System. Publications of the Astronomical Society of the Pacific 123, 423-447.

Harris, A. W. 2002. A New Estimate of the Population of Small NEAs. Bulletin of the American Astronomical Society 34, 835.

Janyes, E. T. 1968. Priori Probabilities. IEEE Transactions On Systems Science and Cybernetics 4, 227-241.

Jenniskens, P., Shaddad, M. H., Numan, D., et al. 2009. The impact and recovery of asteroid $2008 \mathrm{TC}_{3}$. Nature 458, 485-488.

Jurić, M., Ivezić, Ž., Lupton, R. H., et al. 2002. Comparison of Positions and Magnitudes of Asteroids Observed in the Sloan Digital Sky Survey with Those Predicted for Known Asteroids. The Astronomical Journal $124,1776-1787$. 
Kawaguchi, J., Fujiwara, A., Uesugi, T. 2008. Hayabusa - Its technology and science accomplishment summary and Hayabusa-2. Acta Astronautica 62, 639-647.

Kowalski, R. A., Beshore, E. C., Boattini, A., et al. 2008. 2008 TC3. Minor Planet Electronic Circulars 2008-T50.

Kowalski, R. A., Boattini, A., Christesen, E. J., et al. 2014. 2014 AA. Minor Planet Electronic Circulars 2014-A02.

Kozubal, M. J., Gasdia, F. W., Dantowitz, R. F., Scheirich, P., Harris, A. W. 2011. Photometric observations of Earth-impacting asteroid $2008 \mathrm{TC}_{3}$. Meteoritics and Planetary Science 46, 534-542.

Kubica, J., Denneau, L., Grav, T., Heasley, J., Jedicke, R., Masiero, J., Milani, A., Moore, A., Tholen, D., Wainscoat, R. J. 2007. Efficient intraand inter-night linking of asteroid detections using kd-trees. Icarus 189, 151-168.

Larson, S., Brownlee, J., Hergenrother, C., Spahr, T. 1998. The Catalina Sky Survey for NEOs. Bulletin of the American Astronomical Society 30, 1037.

McMillan, R. S. 2000. Spacewatch Survey of the Solar System. NASA STI/Recon Technical Report N 1, 18998.

Milani, A., Chesley, S. R., Chodas, P. W., Valsecchi, G. B. 2002. Asteroid Close Approaches: Analysis and Potential Impact Detection. Asteroids III 55-69. 
Milani, A., Gronchi, G. F., Vitturi, M. D., Knežević, Z. 2004. Orbit determination with very short arcs. I admissible regions. Celestial Mechanics and Dynamical Astronomy 90, 57-85.

Milani, A., Chesley, S. R., Sansaturio, M. E., Tommei, G., Valsecchi, G. B. 2005. Nonlinear impact monitoring: line of variation searches for impactors. Icarus 173, 362-384.

Milani, A., Knežević, Z. 2005. From Astrometry to Celestial Mechanics: Orbit Determination with Very Short Arcs. Celestial Mechanics and Dynamical Astronomy 92, 1-18.

Milani, A., Gronchi, G. F. 2010. Theory of Orbit Determination. Cambridge University Press, Cambridge, UK, 2010.

Muinonen, K., Bowell, E. 1993. Asteroid orbit determination using Bayesian probabilities. Icarus 104, 255-279.

Oszkiewicz, D., Muinonen, K., Virtanen, J., Granvik, M. 2009. Asteroid orbital ranging using Markov-Chain Monte Carlo. Meteoritics and Planetary Science 44, 1897-1904.

Scheirich, P., et al. 2010. The shape and rotation of asteroid $2008 \mathrm{TC}_{3}$. Meteoritics and Planetary Science 45, 1804-1811.

Stokes, G. H., Evans, J. B., Viggh, H. E. M., Shelly, F. C., Pearce, E. C. 2000. Lincoln Near-Earth Asteroid Program (LINEAR). Icarus 148, 21-28.

Stuart, J. S., Binzel, R. P. 2004. Bias-corrected population, size distribution, and impact hazard for the near-Earth objects. Icarus 170, 295-311. 
Virtanen, J., Muinonen, K., Bowell, E. 2001. Statistical Ranging of Asteroid Orbits. Icarus 154, 412-431.

Virtanen, J., Muinonen, K. 2006. Time evolution of orbital uncertainties for the impactor candidate $2004 \mathrm{AS}_{1}$. Icarus 184, 289-301.

Wainscoat, R., Veres, P., Bolin, B., Denneau, L., Jedicke, R., Chastel, S., Micheli, M. 2014. The Pan-STARRS search for near-Earth objects. Asteroids, Comets, Meteors 2014574.

Yamaguchi, T., Yoshikawa, M., Yagi, M., Tholen, D. J. 2011. Entry Dispersion Analysis for the HAYABUSA Spacecraft using Ground-Based Optical Observation. Publications of the Astronomical Society of Japan 63, 979985. 


\begin{tabular}{|c|c|c|c|c|c|c|}
\hline \multirow[t]{2}{*}{ Object } & \multirow{2}{*}{$\begin{array}{c}\text { Number } \\
\text { of obs }\end{array}$} & \multirow{2}{*}{$\begin{array}{c}\text { Arc } \\
\text { length }\end{array}$} & \multirow[t]{2}{*}{ Weights } & \multicolumn{3}{|c|}{ Impact probability } \\
\hline & & & & Jeffreys & Uniform & Population \\
\hline $2008 \mathrm{TC}_{3}$ & 4 & $43 \mathrm{~min}$ & $0.5^{\prime \prime}$ & $6.3 \times 10^{-1}$ & $4.4 \times 10^{-2}$ & $2.4 \times 10^{-2}$ \\
\hline $2008 \mathrm{TC}_{3}$ & 4 & $43 \mathrm{~min}$ & $1.0^{\prime \prime}$ & $6.7 \times 10^{-1}$ & $3.7 \times 10^{-3}$ & $3.5 \times 10^{-3}$ \\
\hline $2008 \mathrm{TC}_{3}$ & 7 & $99 \min$ & $0.5^{\prime \prime}$ & 1.0 & 1.0 & 1.0 \\
\hline $2008 \mathrm{TC}_{3}$ & 7 & $99 \min$ & $1.0^{\prime \prime}$ & $9.7 \times 10^{-1}$ & $9.8 \times 10^{-1}$ & $9.6 \times 10^{-1}$ \\
\hline 2014 AA & 3 & $28 \mathrm{~min}$ & $0.5^{\prime \prime}$ & $8.1 \times 10^{-1}$ & $2.9 \times 10^{-2}$ & $3.1 \times 10^{-2}$ \\
\hline 2014 AA & 3 & $28 \mathrm{~min}$ & $1.0^{\prime \prime}$ & $8.4 \times 10^{-1}$ & $3.3 \times 10^{-3}$ & $8.4 \times 10^{-3}$ \\
\hline 2014 AA & 7 & $69 \mathrm{~min}$ & $0.5^{\prime \prime}$ & 1.0 & 1.0 & 1.0 \\
\hline 2014 AA & 7 & $69 \mathrm{~min}$ & $1.0^{\prime \prime}$ & $9.9 \times 10^{-1}$ & $9.6 \times 10^{-1}$ & $9.0 \times 10^{-1}$ \\
\hline Hayabusa & 3 & $7 \mathrm{~min}$ & $1.0^{\prime \prime}$ & $9.4 \times 10^{-1}$ & $2.7 \times 10^{-3}$ & $2.0 \times 10^{-3}$ \\
\hline Hayabusa & 6 & $34 \mathrm{~min}$ & $1.0^{\prime \prime}$ & $2.4 \times 10^{-1}$ & $1.7 \times 10^{-1}$ & $2.2 \times 10^{-2}$ \\
\hline Hayabusa 2 & 3 & $43 \mathrm{~min}$ & $0.2^{\prime \prime}$ & $2.8 \times 10^{-1}$ & $3.6 \times 10^{-1}$ & $4.2 \times 10^{-1}$ \\
\hline Hayabusa 2 & 3 & $43 \mathrm{~min}$ & $0.5^{\prime \prime}$ & $3.6 \times 10^{-1}$ & $3.3 \times 10^{-2}$ & $7.2 \times 10^{-2}$ \\
\hline Hayabusa 2 & 6 & $26 \mathrm{~h}$ & $0.2^{\prime \prime}$ & $2.4 \times 10^{-13}$ & $2.3 \times 10^{-13}$ & $2.2 \times 10^{-13}$ \\
\hline Hayabusa 2 & 6 & $26 \mathrm{~h}$ & $0.5^{\prime \prime}$ & $1.9 \times 10^{-3}$ & $1.9 \times 10^{-3}$ & $1.8 \times 10^{-3}$ \\
\hline $2004 \mathrm{AS}_{1}$ & 4 & $71 \mathrm{~min}$ & $1.0^{\prime \prime}$ & $2.2 \times 10^{-1}$ & $1.2 \times 10^{-1}$ & $7.8 \times 10^{-2}$ \\
\hline $2004 \mathrm{AS}_{1}$ & 4 & $71 \mathrm{~min}$ & $1.5^{\prime \prime}$ & $1.2 \times 10^{-1}$ & $1.3 \times 10^{-2}$ & $1.1 \times 10^{-2}$ \\
\hline $2004 \mathrm{FU}_{162}$ & 4 & $44 \mathrm{~min}$ & $1.0^{\prime \prime}$ & $3.6 \times 10^{-1}$ & $7.3 \times 10^{-3}$ & $8.7 \times 10^{-4}$ \\
\hline $2004 \mathrm{FU}_{162}$ & 4 & $44 \mathrm{~min}$ & $1.5^{\prime \prime}$ & $5.0 \times 10^{-1}$ & $1.3 \times 10^{-2}$ & $1.0 \times 10^{-3}$ \\
\hline $2015 \mathrm{CV}$ & 4 & $34 \mathrm{~min}$ & $0.5^{\prime \prime}$ & $8.5 \times 10^{-3}$ & $4.7 \times 10^{-6}$ & $9.9 \times 10^{-7}$ \\
\hline $2015 \mathrm{CV}$ & 4 & $34 \mathrm{~min}$ & $1.0^{\prime \prime}$ & $1.7 \times 10^{-1}$ & $1.5 \times 10^{-4}$ & $4.6 \times 10^{-5}$ \\
\hline $2015 \mathrm{BU}_{92}$ & 3 & $36 \mathrm{~min}$ & $0.5^{\prime \prime}$ & $1.7 \times 10^{-1}$ & $7.2 \times 10^{-5}$ & $3.5 \times 10^{-10}$ \\
\hline $2015 \mathrm{BU}_{92}$ & 3 & $36 \mathrm{~min}$ & $1.0^{\prime \prime}$ & $4.8 \times 10^{-1}$ & $4.0 \times 10^{-4}$ & $8.5 \times 10^{-8}$ \\
\hline
\end{tabular}

Table 2: Impact probabilities for the examples considered in Sec. 6 for different choices of $f_{\text {prior }}$, different number of tracklets, and different data weights. 


\begin{tabular}{|lc|ccc|}
\hline Object & Weights & \multicolumn{3}{|c|}{$p$-value } \\
& & Jeffreys & Uniform & Population \\
\hline $2004 \mathrm{AS}_{1}$ & $1.0^{\prime \prime}$ & $3.1 \times 10^{-5}$ & $2.6 \times 10^{-2}$ & $1.4 \times 10^{-1}$ \\
$2004 \mathrm{AS}_{1}$ & $1.5^{\prime \prime}$ & $4.0 \times 10^{-4}$ & $2.2 \times 10^{-1}$ & $3.9 \times 10^{-1}$ \\
\hline $2015 \mathrm{CV}$ & $0.5^{\prime \prime}$ & $5.0 \times 10^{-5}$ & $4.9 \times 10^{-1}$ & $4.2 \times 10^{-1}$ \\
$2015 \mathrm{CV}$ & $1.0^{\prime \prime}$ & $1.3 \times 10^{-5}$ & $4.9 \times 10^{-1}$ & $4.2 \times 10^{-1}$ \\
\hline $2015 \mathrm{BU}_{92}$ & $0.5^{\prime \prime}$ & $1.1 \times 10^{-5}$ & $9.1 \times 10^{-1}$ & $8.8 \times 10^{-1}$ \\
$2015 \mathrm{BU}_{92}$ & $1.0^{\prime \prime}$ & $2.3 \times 10^{-6}$ & $9.1 \times 10^{-1}$ & $8.9 \times 10^{-1}$ \\
\hline
\end{tabular}

Table 3: $p$-values for some of the examples considered in Sec. 6 for different choices of $f_{\text {prior }}$ and different data weights. 